\title{
Evaluation of the in vitro Function of Platelet Concentrates from Pooled Buffy Coats or Apheresis
}

\author{
Sarah Anna Fiedler ${ }^{a} \quad K^{2}$ laus Boller ${ }^{b} \quad$ Ann-Christine Junker ${ }^{a} \quad$ Christel Kamp $^{c}$ \\ Anneliese Hilger ${ }^{a}$ Wolfgang Schwarz ${ }^{a}$ Rainer Seitz ${ }^{a} \quad$ Ursula Salge-Bartels $^{a}$

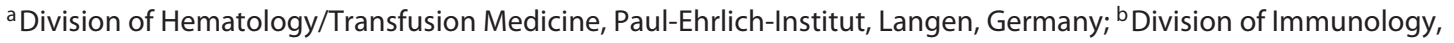 \\ Paul-Ehrlich-Institut, Langen, Germany; ' Division of Microbiology, Paul-Ehrlich-Institut, Langen, Germany
}

\section{Keywords}

Platelet concentrates · Platelet function · Platelet

aggregation · Shear stress · Fluidic system

\begin{abstract}
Background: Platelet concentrates play an important role in transfusion medicine. Their short lifespan and lack of robustness require efforts to ensure adequate product quality. In this study, we compared the in vitro quality of the main concentrate types, pooled platelet concentrate (PPC) from whole blood donations, and platelet concentrate from single-donor apheresis (APC). Methods: Twenty PPCs and 20 APCs prepared in plasma were analyzed on days 2,4 , and 7 of storage. Variables related to metabolism, degranulation, platelet aggregation, P-selectin expression, and annexin $\mathrm{V}$ binding were analyzed. Morphology was assessed by transmission electron microscopy of ultrathin sections. A microfluidic device was applied to test the effects of shear stress on platelet function. Results: The metabolic parameters indicated stable storage conditions throughout the 7-day period. The resting discoid form was the prevailing morphology on days 2 and 4 in the PPCs and APCs. Chemokine release and receptor shedding of soluble P-selectin and soluble CD40L equally increased in PPCs and APCs. Aggregation responses to ADP and collagen were heterogeneous, with marked losses in collagen responsiveness on day 4 in individual concentrates. Baseline expression of P-selectin in PPCs and APCs was low, and inducibility of P-selectin was
\end{abstract} increase product reliability.

\section{Introduction} siveness and stability were found with platelets from PPCs and APCs. Conclusions: Platelets from PPCs and APCs showed similar in vitro function and stability parameters. However, platelet concentrates presented a high variability and individual concentrates an impaired functional capability. Identifying the factors contributing to this would help

(c) 2020 S. Karger AG, Basel

Platelet (PLT) concentrates (PCs) are irreplaceable therapeutics and their clinical benefit is clear. However, transfusion refractoriness was reported as an encountered problem occurring in $27 \%$ of patients [1]. Underlying causes can be patient-associated factors, such as fever, medication, sepsis, or patient anti-PLT antigen (HPA, human PLT antigen) antibodies, or can otherwise be due to the increased consumption of PLTs within the scope of the desired hemostasis $[2,3]$. In contrast, PLT refractoriness can be product-related and associated with PLT-inherent functional variations due to the habitual and physiological conditions of the donors [4]. The manufacturing process may also impact PLT functionality [5]. In Germany, two types of PC production are applied: preparation by pooling of PLTs from whole blood buffy coats (pooled platelet concentrate, PPC) and preparation by 
single-donor apheresis (APC). In clinical use, the decision for either PC type is not clearly defined. Therefore, there is some uncertainty surrounding the quality and equivalence of both PC types. The transfusion of selected single-donor APCs in the case of individual immunological requirements is beyond question; however, in cases which do not require special donor selection, quality and usage of the different products are controversially discussed [5-9].

In order to obtain additional information on PLT stability for both types of manufacturing, we implemented a fluidic test system to address the effect of shear forces on PLT integrity in vitro. In earlier studies, researchers reported the differential effects of shear forces on PLT activation status. Depending on the extent and duration, exposure to shear stress can either cause a loss of responsiveness to external stimulation [10] or have a sensitizing effect [11]. Whether different manufacturing methods have an impact on PLT stability and robustness has not been studied so far but could provide valuable information for the in vitro evaluation of PLT integrity.

In this study, we carried out a comparative analysis with PPC and APC by applying a comprehensive panel of established methods to determine the metabolic and functional parameters, as well as PLT morphology. In addition, we examined the challenging effect of wall shear stress to address PLT robustness and functional capabilities.

\section{Materials and Methods}

\section{Platelet Concentrates}

We conducted this study in accordance with German legislation and according to the "Helsinki Ethical Principles." Forty PCs (blood group A, rhesus+) were purchased from two different blood banks (source A and B), each providing 10 PPCs and 10 APCs on day 1 of production. In order to minimize effects from matrix heterogeneity we chose PLT preparations in plasma. PPCs were prepared from four or five buffy coats using the OrbiSac System (Terumo, Eschborn, Germany) in CPD (citrate, phosphate, dextrose) anticoagulated plasma. APCs were collected in ACD-A (acid citrate dextrose, adenine) and plasma by use of the Trima Accel ${ }^{\circledR}$ automated blood collection system (Terumo). PCs were stored at $22^{\circ} \mathrm{C}\left( \pm 2^{\circ} \mathrm{C}\right)$ under continuous agitation $(60 \mathrm{rpm}$, Helmer Platelet Incubator; Fresenius, Bad Homburg, Germany) for 7 days. All samples were drawn under aseptic conditions.

\section{Visual Inspection, PLT Count, pH, and Metabolic Variables}

Swirling was regularly assessed and classified as 0 (none), 1 (some), or 2 (strong). PLT counts were estimated microscopically (Axiovert; Zeiss) through the use of a Neubauer chamber and dilution with ThromboCount ${ }^{\circledR}$ (Bioanalytic, Umkirch, Germany).

$\mathrm{pH}$ values were measured at $22^{\circ} \mathrm{C}\left( \pm 2^{\circ} \mathrm{C}\right)$ immediately after sampling using a laboratory $\mathrm{pH}$ meter (SI Analytics, Mainz, Germany). Glucose concentrations were determined using the ACCU-CHEK Aviva System (Roche Diagnostics, Mannheim, Germany). Lactate content was estimated by applying an optical test kit (Sigma-Aldrich, Taufkirchen, Germany).

\section{Sterility Testing}

We performed sterility tests initially and on day 7 of storage with 5-mL samples under aerobic and anaerobic culture conditions (BacT/ALERT ${ }^{\circledR}$; Biomérieux, Nürtingen, Germany). All samples tested negative after 14 days of incubation.

\section{Transmission Electron Microscopy}

We processed the original samples from undiluted PC using a two-step fixation procedure. In the first step, $0.2 \%$ glutaraldehyde was added and incubated for $60 \mathrm{~min}$ at room temperature, and after centrifugation at $400 \mathrm{~g}$ for $10 \mathrm{~min}, 2.5 \%$ glutaraldehyde was added and incubated for another $60 \mathrm{~min}$ [12]. The PLT pellets were further processed for transmission electron microscopy (TEM) following standard procedures [13]. Briefly, ultrathin sections were viewed at 1,100× magnification in a Zeiss EM 902 electron microscope (Zeiss) equipped with a TRS digital camera and ITEM software (EMSIS, Münster, Germany). Five representative micrographs per sample were quantitatively evaluated to differentiate the PLTs according to their shapes (i.e., discoid, spheroid, apoptotic, or ballooned).

\section{Flow Cytometry}

Flow cytometry (FCM) was performed on an EPICS-XL instrument (Beckman Coulter, Krefeld, Germany) by evaluating at least 10,000 events in the PLT gate or a volume corresponding to a 10$\mu \mathrm{L}$ sample by application of Flow Count Fluorospheres ${ }^{\mathrm{TM}}$ (Beckman Coulter). The residual erythrocyte and leucocyte numbers were assessed on the first day of preparation by FCM as previously described [14], and PLT analysis was performed according to protocols described earlier [15]. Briefly, the PLTs were double stained with fluorescein isothiocyanate (FITC)-conjugated antiCD41 and the phycoerythrin-conjugated PLT activation marker anti-CD62-P (P-selectin; Beckman Coulter), either with non-stimulated PLT or after stimulation with $10 \mu \mathrm{mol} / \mathrm{L}$ TRAP-6 (thrombin receptor activating peptide, SFLLRN; Bachem, Bubendorf, Switzerland) for $10 \mathrm{~min}$ at $37^{\circ} \mathrm{C}$ before staining. After the addition of $800 \mu \mathrm{L}$ FCM buffer (PBS, 1\% BSA, 0.09\% sodium azide; Sigma), the measurements were carried out within $20 \mathrm{~min}$. Phosphatidylserine (PS) expression was determined by annexin V-FITC binding as described previously [16]. As a viability stain, calcein-AM (calcein acetoxymethyl ester; PromoCell, Heidelberg, Germany) was applied. Suspensions of $1-2 \times 10^{6}$ PLTs/100 $\mu$ L HBS (Hepes buffered saline, $10 \mathrm{mmol} / \mathrm{L}$ Hepes (4-(2-Hydroxyethyl)piperazine-1-ethanesulfonic acid sodium salt), $150 \mathrm{mmol} / \mathrm{L}$ sodium chloride, $0.1 \%$ glucose, $\mathrm{pH} 7.4)$ were incubated with $0.5 \mu \mathrm{mol} / \mathrm{L}$ calcein-AM and $10 \mu \mathrm{L}$ anti-CD41-PC5 (phycoerythrin-cyanin 5; Beckman Coulter) for $15 \mathrm{~min}$ at $37^{\circ} \mathrm{C}$ in the dark. After the addition of FCM buffer, the samples were analyzed immediately.

\section{PLT Aggregation by Light Transmission Aggregometry}

Light transmission aggregometry measurements were performed with an aggregometer (Chrono-log series 490; Probe \& go Labordiagnostica, Lemgo, Germany) with PLT numbers adjusted to $3 \times 10^{8} / \mathrm{mL}$ with $\mathrm{AB}$ plasma (Blood Donation Center Darmstadt, Germany). Agonists were adenosine $5^{\prime}$ diphosphate (ADP, 10 $\mu \mathrm{mol} / \mathrm{L}$; Probe $\&$ go $),$ TRAP-6 $(100 \mu \mathrm{mol} / \mathrm{L}), 2 \mu \mathrm{g} / \mathrm{mL}$ collagen, and $1 \mathrm{mmol} / \mathrm{L}$ arachidonic acid (AA; Haemochrom Diagnostica, Essen, Germany). Samples with an aggregation response to 1 $\mathrm{mmol} / \mathrm{L}$ AA below $2 \%$ were classified as nonresponsive, while samples with aggregation between 2 and $7 \%$ were classified as low responsive.

Extent of Shape Change and Hypotonic Shock Resistance

Extent of shape change (ESC) and hypotonic shock resistance (HSR) were assessed according to Holme et al. [17] with $3 \times 10^{8}$ 
PLTs/mL in AB plasma using an optical aggregometer (Chrono$\log$ ) with manual calculation or through the use of the automated SPA-2000 device (Chrono-log).

\section{Rotational Thromboelastometry}

Rotational thromboelastometry $\left(\right.$ Rotem $^{\circledR}$ delta Hemostasis Analyzer; Werfen, Munich, Germany) was applied to test the PLT interaction with the coagulation system in a global clotting assay system on days 2 and 4 of storage. PCs adjusted to $3 \times 10^{8}$ PLTs/ $\mathrm{mL}$ in $\mathrm{AB}$ plasma were recalcified with starTEM ${ }^{\mathrm{TM}}$ reagent according to the supplier's instructions.

\section{Effect of Shear Stress on PLT Adhesion and Activation}

To test the PLT behavior under conditions of elevated shear stress we performed flow chamber experiments according to earlier methods $[18,19]$. The flow experiments were performed in the absence of red blood cells in order to preclude the addition of further variables from external sources. An ibidi perfusion system (Martinsried, Germany) consisting of a computer-controlled pump unit and collagen-coated $\mu$-slides VI 0.4, (mouse collagen IV, \#356233; Corning) was applied according to the supplier's instructions. Ten $\mathrm{mL}$ of pre-warmed PCs with $3 \times 10^{8}$ PLTs $/ \mathrm{mL}, 37^{\circ} \mathrm{C}\left( \pm 0.5^{\circ} \mathrm{C}\right)$, were unidirectionally pumped through the channels applying a wall shear rate of $1,700 / \mathrm{s}$ for 4 min. The shear rate of $1,700 / \mathrm{s}$ corresponded to a wall shear stress of $20 \mathrm{dyn} / \mathrm{cm}^{2}$ calculated according to the equation provided in the ibidi application note $\left(\tau\left[\mathrm{dyn} / \mathrm{cm}^{2}\right]=1.761 \times\right.$ flow rate $[\mathrm{mL} /$ $\mathrm{min}]$ ) [20]. For the PCs in plasma, we estimated a viscosity of 1.2 $\mathrm{mPa}$ s at $37^{\circ} \mathrm{C}$ using a Brookfield DV-II viscometer (Brookfield Ametek ${ }^{\circledR}$, Lorch, Germany). Assays comprised static and shear conditions with and without $5 \mu \mathrm{mol} / \mathrm{L}$ TRAP-6. In a first series, comprising 10 PPCs and 10 APCs, we analyzed PLT adhesion after PLT staining with an anti-CD41-FITC antibody (Beckman Coulter) for $45 \mathrm{~min}$. Following this, we evaluated the slides using fluorescence microscopy (Axiovert 200; Zeiss) at 630-fold magnification. We then examined ten photographs taken of the representative channel areas to find out the number of adherent PLTs. In a second series (10 PPCs and 10 APCs), we analyzed the way in which the applied challenge of shear stress and TRAP-6 affected the non-adherent PLT fraction. To this end, we performed cellular function assays immediately after challenge, evaluating PLT CD62-P expression and PLT aggregation in response to ADP $(10 \mu \mathrm{mol} / \mathrm{L})$, collagen $(2 \mu \mathrm{g} / \mathrm{mL})$, and TRAP-6 $(100 \mu \mathrm{mol} / \mathrm{L})$, and by thromboelastometry after recalcification. Furthermore, cell-free supernatant plasma was prepared to assay plasma analytes.

\section{Plasma Analytes}

PC supernatant plasma was prepared using centrifugation $(2,500 \mathrm{~g}, 10 \mathrm{~min}$, room temperature $)$ and stored frozen at $-30^{\circ} \mathrm{C}$ until analysis. We then applied ELISAs to determine human CCL5/ RANTES (regulated on activation, normal T cell expressed and secreted), CXCL-7/NAP-2 (neutrophil activating protein-2), soluble (s)P-selectin (Bio-techne, Wiesbaden, Germany), soluble (s) CD40L (eBioscience, Thermo Fisher Schwerte, Germany), IL-6 (Diaclone; Hölzel, Cologne, Germany), complement factors C3a des-Arg (Progen, Heidelberg, Germany), and C5b-9 (BD Bioscience, Heidelberg, Germany). Lactate dehydrogenase (LDH) activity in plasma was assayed at $37^{\circ} \mathrm{C}$ using the commercial LDH L-P Kit adapted for application in a BCS-XP analyzer system (Siemens Healthcare Diagnostics, Eschborn, Germany). We applied the Enz1-CAL ${ }^{\mathrm{TM}}$ reference to estimate the plasma LDH concentrations using the slope ratio method with the CombiStats ${ }^{\mathrm{TM}}$ program (European Directorate for the Quality of Medicines and Healthcare, Strasbourg, France).

\section{Statistical Evaluation}

We evaluated the differences in measured parameters between PPCs and APCs using the nonparametric Wilcoxon rank-sum test (Mann-Whitney test) using R statistics software (https://www. r-project.org/). Differences in the distribution of morphologic types between PPC and APC were studied with the $\chi^{2}$ test of homogeneity using GraphPad Prism software (https://www.graphpad.com/scientific-software/prism/). Since this was an explorative study, we made no adjustments in the significance levels to correct for multiple testing. Overall, we chose a significance level of 0.01 and considered $p$ values $<0.01$ as statistically significant.

\section{Results}

\section{PC Specifications}

All PCs complied with the specifications according to the German guidelines [21] regarding PLT and residual cell numbers. PLT numbers did not change significantly during storage until day 7. Sterility was confirmed for all PCs (online suppl. Table S1; for all online suppl material, see www.karger.com/doi/10.1159/000504917).

\section{Parameters of PTL Metabolism and Stability}

Indicators of a balanced energy metabolism differed only marginally between PPCs and APCs, such as glucose consumption (Fig. 1A) and lactate accumulation (Fig. 1B). Due to the manufacturing protocols glucose concentrations in PPCs were $2 \mathrm{mmol} / \mathrm{L}$ higher than in APCs. PPCs started with median concentrations (1st and 3rd quartiles) of $20.2 \mathrm{mmol} / \mathrm{L}(19.4 ; 20.6)$ compared to APCs with $18.2 \mathrm{mmol} / \mathrm{L}(16.3 ; 18.9)$ on day 2 . However, the rates of glucose consumption were identical for both sources and types (Fig. 1A). Median values of lactate concentrations on day 2 were higher in PPCs than in APCs (1st and 3rd quartiles): PPCs, $4.5 \mathrm{mmol} / \mathrm{L}$ (4.0; 5.1); APCs, $2.8 \mathrm{mmol} / \mathrm{L}$ $(2.5 ; 3.1)$, but the lactate production rates did not differ between the preparation types and sources (Fig. 1B). Both parameters indicated equivalent metabolic turnover during the entire storage period which is supported by almost constant plasma $\mathrm{pH}$ values (Table 1). The "swirling" test, a point of care test, and also HSR and ESC are established tests for general PLT "fitness"; all three tests indicated a high vitality for PPCs and APCs (Table 1). A strong swirling (score 2) was found with all PCs throughout the 7-day period. In line, equal HSR and ESC values were measured in PPCs and APCs without change under storage. However, regardless of the PC type, individual PCs showed broad variations in HSR values ranging from 20 to $70 \%$ on day 2. LDH concentrations did not significantly differ between PPCs and APCs on day 2: PPCs, $131.5 \mathrm{IU} / \mathrm{L}$ (114; 157.3); APCs, $142.0 \mathrm{IU} / \mathrm{L}(125.5 ; 155.8)$, and remained nearly unchanged until day 4 of storage (Table 1 ). The slight increase in APCs seen on day 7 apparently is due to individual outliers. In line with this is the high calcein 
Fig. 1. Glucose consumption and lactate production in PPCs and APCs from two different sources (source A and B) during storage. Values were normalized to $10^{12}$ PLTs/L. Glucose values (A) steadily declined and, conversely, lactate concentrations (B) increased equally in the course of storage for both sources and types. Given are means \pm SD. $n=10$ PPCs (black symbols), $n=10$ APCs (gray symbols) of source A (dots) and source B (triangles).
(A)

\section{Glucose}

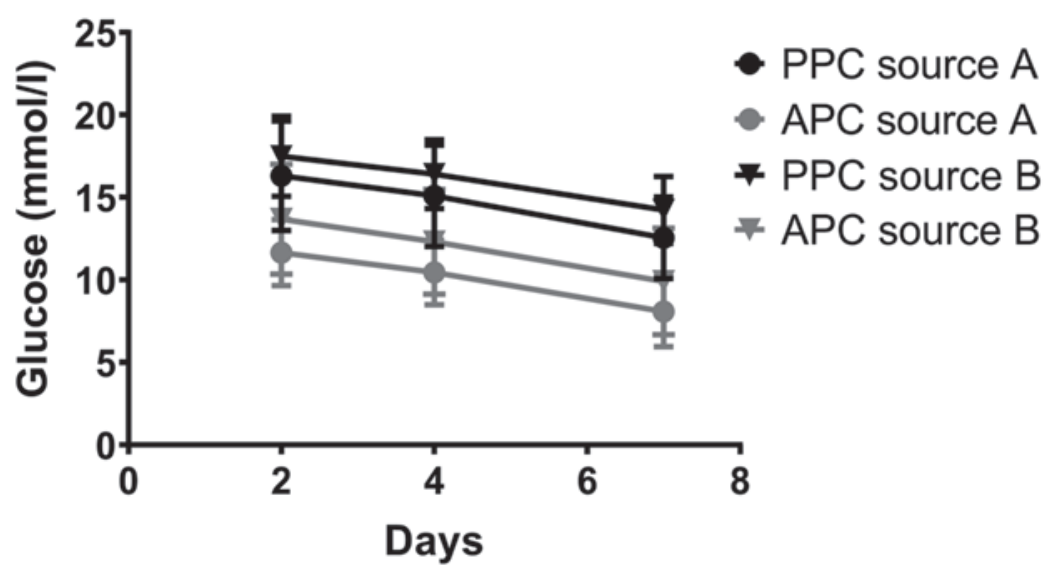

(B)

Lactate

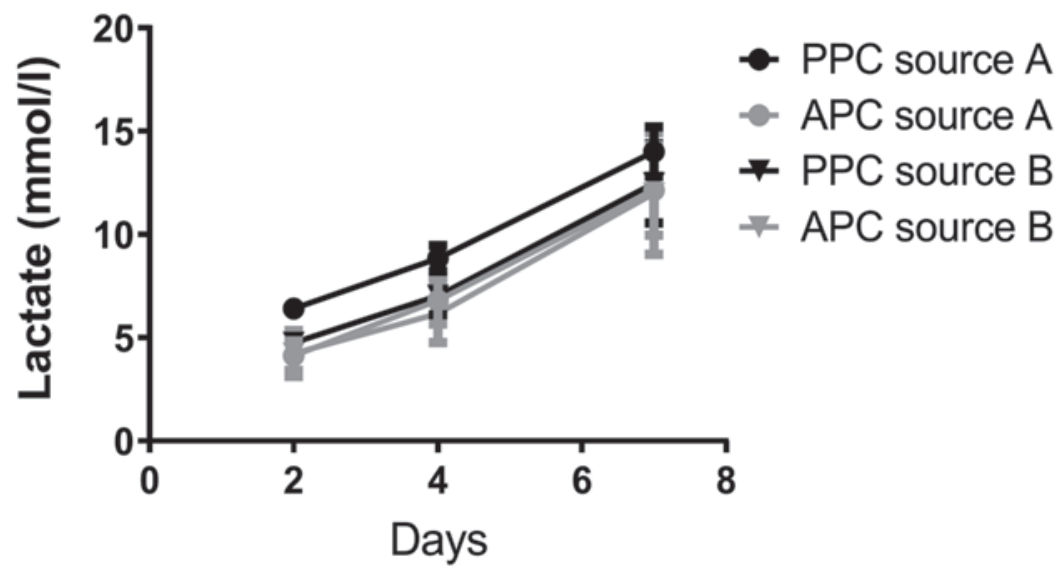

staining ability, a marker of cell vitality, which showed constant intensities until day 4 and very few losses on day 7 (Table 1).

\section{Markers of PLT Activation in Plasma}

With respect to plasma-related effects, foreign surfaces are well-known activators of the complement system via the alternative pathway triggered by complement factor $\mathrm{C} 3 \mathrm{a}$, a process that might also occur during blood manufacturing and PLT storage. Our data (Table 1) initially showed moderately elevated C3a des-Arg values that steadily increased until day 7 , reaching values as high as $6,600 \mathrm{ng} / \mathrm{mL}$ plasma (normalized to $10^{9} \mathrm{PLT} / \mathrm{mL}$ ) in individual PCs. The soluble terminal complex C5b-9, which is indicative of cellular assaults, increased only modestly until day 4 , thus suggesting a minor impact on cell vitality.
Shedded extracellular receptor domains, such as sPselectin and SCD40L (Table 1), were determined as markers of a preceding PLT activation. Initial sP-selectin levels were low with median values ( 1 st and 3 rd quartiles) of 55 $\mathrm{ng} / \mathrm{mL}(43 ; 70)$ in PPCs, and $43 \mathrm{ng} / \mathrm{mL}(35 ; 55)$ in APCs. During storage an about 2 -fold increase was observed on day 7 in both PC types. Only on day 4 were the sP-selectin levels slightly, but significantly, higher in PPCs than in APCs: PPCs, $95 \mathrm{ng} / \mathrm{mL}(86 ; 111)$ vs. APCs, $75 \mathrm{ng} / \mathrm{mL}$ (62; $86), p<0.001)$. A relevance of these slight differences, which were not found on day 7 , seems implausible.

This is in line with sCD40L data which, like sP-selectin, increased during storage but did not significantly differ between PPCs and APCs. The plasma values of the chemokines CXCL7 and RANTES that indicate $\alpha$-granule release, steadily increased during storage time by 3 - to 
Table 1. Storage variables related to metabolism, vitality, and activation status

\begin{tabular}{|c|c|c|c|}
\hline Parameters & & $\operatorname{PPC}(n=20)$ & $\operatorname{APC}(n=20)$ \\
\hline \multirow[t]{3}{*}{$\mathrm{LDH}, \mathrm{IU} / \mathrm{l}^{\#}$} & Day 2 & $131.5(114 ; 157.3)$ & $142.0(125.5 ; 155.8)$ \\
\hline & Day 4 & $143.5(132.3 ; 158.5)$ & $154.0(129.0 ; 197.3)$ \\
\hline & Day $7^{\star *}$ & $145.5(105.3 ; 171.5)$ & $183.0(145.3 ; 229.0)$ \\
\hline \multirow[t]{3}{*}{$\mathrm{pH}\left(22^{\circ} \mathrm{C}\right)$} & Day 2 & $7.58(7.52 ; 7.62)$ & $7.59(7.54 ; 7.62)$ \\
\hline & Day 4 & $7.64(7.58 ; 7.68)$ & $7.60(7.57 ; 7.62)$ \\
\hline & Day 7 & $7.49(7.45 ; 7.54)$ & $7.45(7.38 ; 7.50)$ \\
\hline \multirow[t]{3}{*}{ HSR, \% } & Day 2 & $50.9(40.4 ; 71.8)$ & $62.2(39.2 ; 73.1)$ \\
\hline & Day 4 & $50.1(41.4 ; 63.6)$ & $47.2(36.2 ; 62.2)$ \\
\hline & Day 7 & $56.3(38.5 ; 78.2)$ & $53.4(38.9 ; 71.9)$ \\
\hline \multirow[t]{3}{*}{ ESC, \% } & Day 2 & $12.9(10.1 ; 15.6)$ & $12.2(9.3 ; 16.3)$ \\
\hline & Day 4 & $12.4(11.3 ; 15.2)$ & $13.0(10.4 ; 17.1)$ \\
\hline & Day 7 & $12.0(10.7 ; 14.9)$ & $15.1(11.1 ; 17.6)$ \\
\hline \multirow[t]{3}{*}{ PLT calcein MFI } & Day 2 & $140(112 ; 153)$ & $88(68 ; 153)$ \\
\hline & Day 4 & $134(99 ; 185)$ & $101(76 ; 192)$ \\
\hline & Day 7 & $118(90 ; 149)$ & $93(60 ; 124)$ \\
\hline \multirow[t]{3}{*}{ C3a des-Arg, ng/mL } & Day 2 & $443(290 ; 674)$ & $466(143 ; 890)$ \\
\hline & Day 4 & $1,372(934 ; 2,149)$ & $1,672(1,058 ; 2,213)$ \\
\hline & Day 7 & $6,559(5,817 ; 7,170)$ & $6,682(4,676 ; 8,131)$ \\
\hline \multirow[t]{3}{*}{ C5b-9, ng/mL } & Day 2 & $145(103 ; 211)$ & $140(110 ; 239)$ \\
\hline & Day 4 & $400(259 ; 517)$ & $416(282 ; 503)$ \\
\hline & Day 7 & $373(233 ; 440)$ & $371(266 ; 558)$ \\
\hline \multirow[t]{3}{*}{ sP-selectin, ng/mL $L^{\#}$} & Day 2 & $55(43 ; 70)$ & $43(35 ; 55)$ \\
\hline & Day $4^{* * *}$ & $95(86 ; 111)$ & $75(62 ; 86)$ \\
\hline & Day 7 & $124(95 ; 132)$ & $93(75 ; 112)$ \\
\hline \multirow[t]{3}{*}{$\mathrm{sCD} 40 \mathrm{~L}, \mathrm{ng} / \mathrm{mL}^{\#}$} & Day 2 & $4.09(2.50 ; 4.73)$ & $2.78(2.13 ; 3.26)$ \\
\hline & Day 4 & $6.94(4.90 ; 8.09)$ & $5.24(3.47 ; 6.59)$ \\
\hline & Day 7 & $6.96(5.90 ; 9.98)$ & $6.92(5.02 ; 9.11)$ \\
\hline \multirow[t]{3}{*}{ CXCL7/NAP-2, ng/mL } & Day 2 & $1,876(1,485 ; 2,423)$ & $1,200(940 ; 1,572)$ \\
\hline & Day 4 & $3,890(3,175 ; 4,985)$ & $2,666(1,795 ; 3,853)$ \\
\hline & Day 7 & $5,818(4,993 ; 6,607)$ & $4,774(3,448 ; 6,042)$ \\
\hline \multirow[t]{3}{*}{ RANTES, ng/mL } & Day 2 & $56(39 ; 83)$ & $50(42 ; 60)$ \\
\hline & Day 4 & $127(108 ; 165)$ & $109(88 ; 143)$ \\
\hline & Day 7 & $243(195 ; 290)$ & $233(192 ; 305)$ \\
\hline
\end{tabular}

Values are given as medians (with 1st and 3rd quartiles). " Plasma analyte concentrations were normalized to $10^{9} \mathrm{PLTs} / \mathrm{mL} .{ }^{* *} p<0.01{ }^{* * *} p<0.001$, significant differences between PPCs and APCs.

4-fold of initial concentrations, irrespective of the PC type. PAF, another inflammatory factor, was only found in low amounts in PPCs and APCs on day 2: PPCs, 278 $\mathrm{pg} / \mathrm{mL}(179 ; 426)$ vs. APCs, $221 \mathrm{pg} / \mathrm{mL}$ (157; 332). PAF values remained constant during the whole storage period. Overall, no significant differences were observed between PPCs and APCs, yet considerable variations between individual PCs were evident in both groups.

\section{PLT Morphology}

The structural integrity of PLTs was determined after 2 and 4 days of storage, which is the authorized storage period in Germany. We prepared ultra-thin sections of each sample and evaluated micrographs of five different regions by classifying the cellular structure as either "discoid," "spheroid," "apoptotic," or "ballooned." PPC and APC preparations displayed similar morphologies on days 2 and day 4 of storage (Fig. 2). Quantification results from day 4 showed that intact, not activated discoid cells made up the largest proportion, whereas only a minor fraction was activated and displayed a ballooned (approximately 6\%) or even a clear apoptotic appearance (approximately $1 \%$; Table 2 ). The distributions between different PLT shapes showed a significant difference between PPCs and APCs $(p<0.0001)$ which can largely be attributed to a shift from spheroid to discoid PLTs in 
Fig. 2. PLT morphology evaluated by TEM imaging. Representative images depicting PLT structure on day 2 (top) and day 4 (bottom) of storage; PPC (left) and APC (right). Original magnification, $\times 1,100$. Scale bars, $5 \mu \mathrm{m}$.

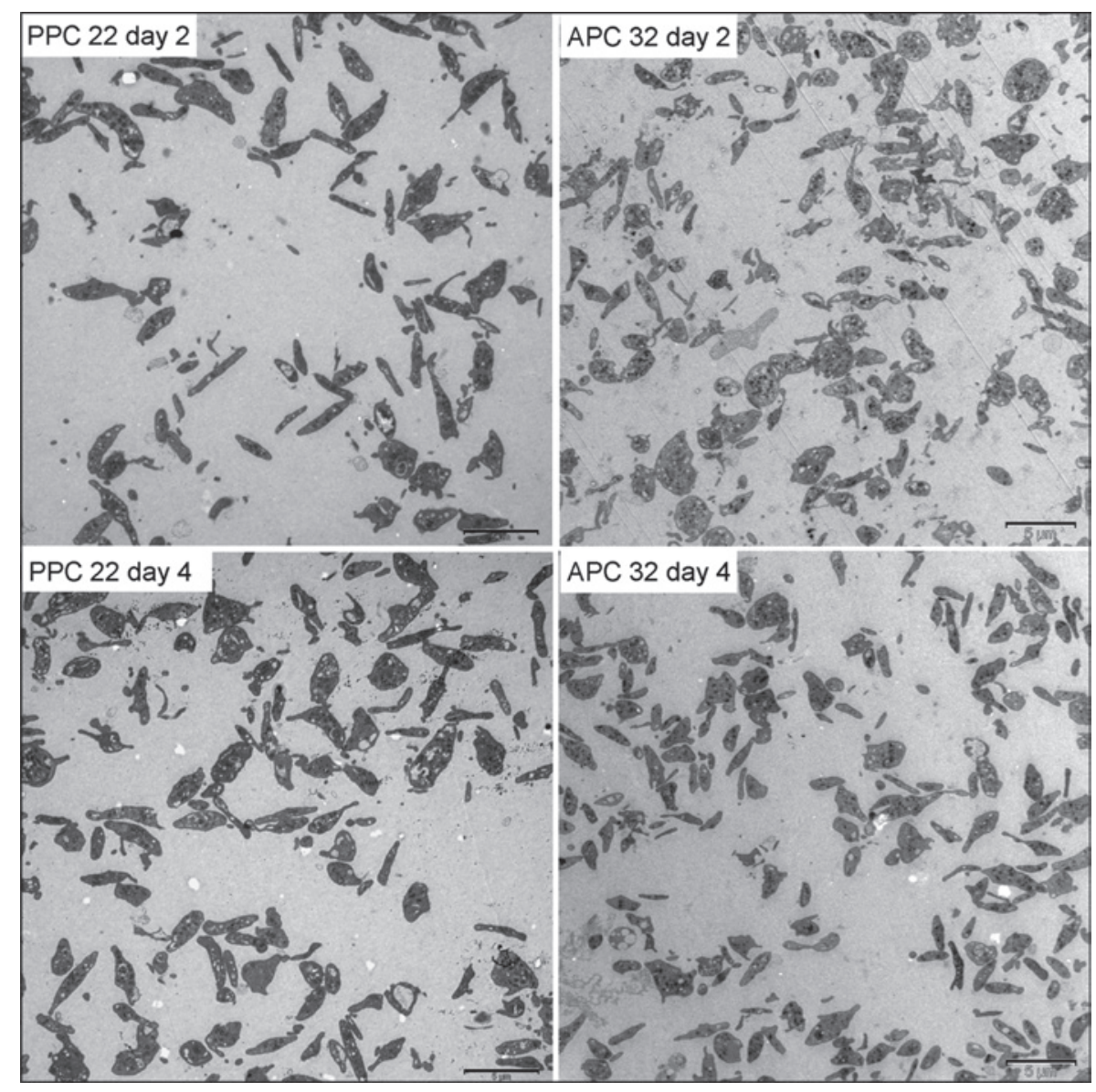

Table 2. Platelet morphology evaluated by TEM imaging on day 4 of storage

\begin{tabular}{lrrrr}
\hline PLT morphology & $\begin{array}{l}\text { PPC } \\
(n=20)\end{array}$ & $\begin{array}{l}\text { PPC, } \\
\%\end{array}$ & $\begin{array}{l}\text { APC } \\
(n=20)\end{array}$ & \multicolumn{1}{c}{$\begin{array}{l}\text { APC, } \\
\%\end{array}$} \\
\hline Apoptotic & 92 & 1.2 & 53 & 0.8 \\
Discoid**** & 5,639 & 76.5 & 7,089 & 82.0 \\
Spheroid & 1,114 & 15.1 & 1,029 & 11.9 \\
Ballooned & 451 & 6.2 & 474 & 5.5 \\
\hline Sum & 7,373 & 100 & 8,645 & 100 \\
\hline
\end{tabular}

Values are cumulative numbers from individual preparations of the PPCs and APCs which showed significant differences in their distributions under a $\chi^{2}$ test of homogeneity. ${ }^{* * * *} p<0.0001$.

APCs compared to PPCs. However, it cannot be ruled out that the higher platelet concentrations of APCs during sampling could have some impact on clear assignment.

\section{PLT Aggregation and Thromboelastometry}

The aggregation response was induced through the addition of $10 \mu \mathrm{mol} / \mathrm{L}$ ADP (Fig. 3A), $2 \mu \mathrm{g} / \mathrm{mL}$ collagen, (Fig. 3B), and $100 \mu \mathrm{mol} / \mathrm{L}$ TRAP-6 (not shown). Responses were strongest on day 2 but varied widely between in- dividual PCs. In PPCs and APCs, the median aggregation response to ADP continuously declined from about $70 \%$ on day 2 to approximately $25 \%$ on day 7 (Fig. $3 \mathrm{~A}$ ). The response to collagen was found to be more vulnerable and variable, i.e. median values declined from $70 \%$ on day 2 to $5 \%$ on day 7 . It was noted that a few preparations did not show adequate responses already on day 2 (Fig. 3B). However, the addition of TRAP- 6 elicited almost a 100\% response rate in all PCs (not shown). No significant differences were found between the PPCs and APCs.

Thromboelastometry was applied to determine the contribution of PCs to clot formation and clot firmness. Clotting time was generally not affected by PLTs at any time point, whereas clot formation time and maximal clot firmness showed clear PLT dependent increases (online suppl. Table S2). Storage-dependent changes and differences between PPCs and APCs were not evident in this assay.

\section{PLT P-Selectin and PS Expression}

$\mathrm{P}$-selectin (CD62-P) expression was measured to assess preparation and storage-dependent activation (Fig. 3C, D). No significant differences were observed between PPCs and APCs throughout the 7-day storage period. For both types, baseline $\mathrm{P}$-selectin expression levels 


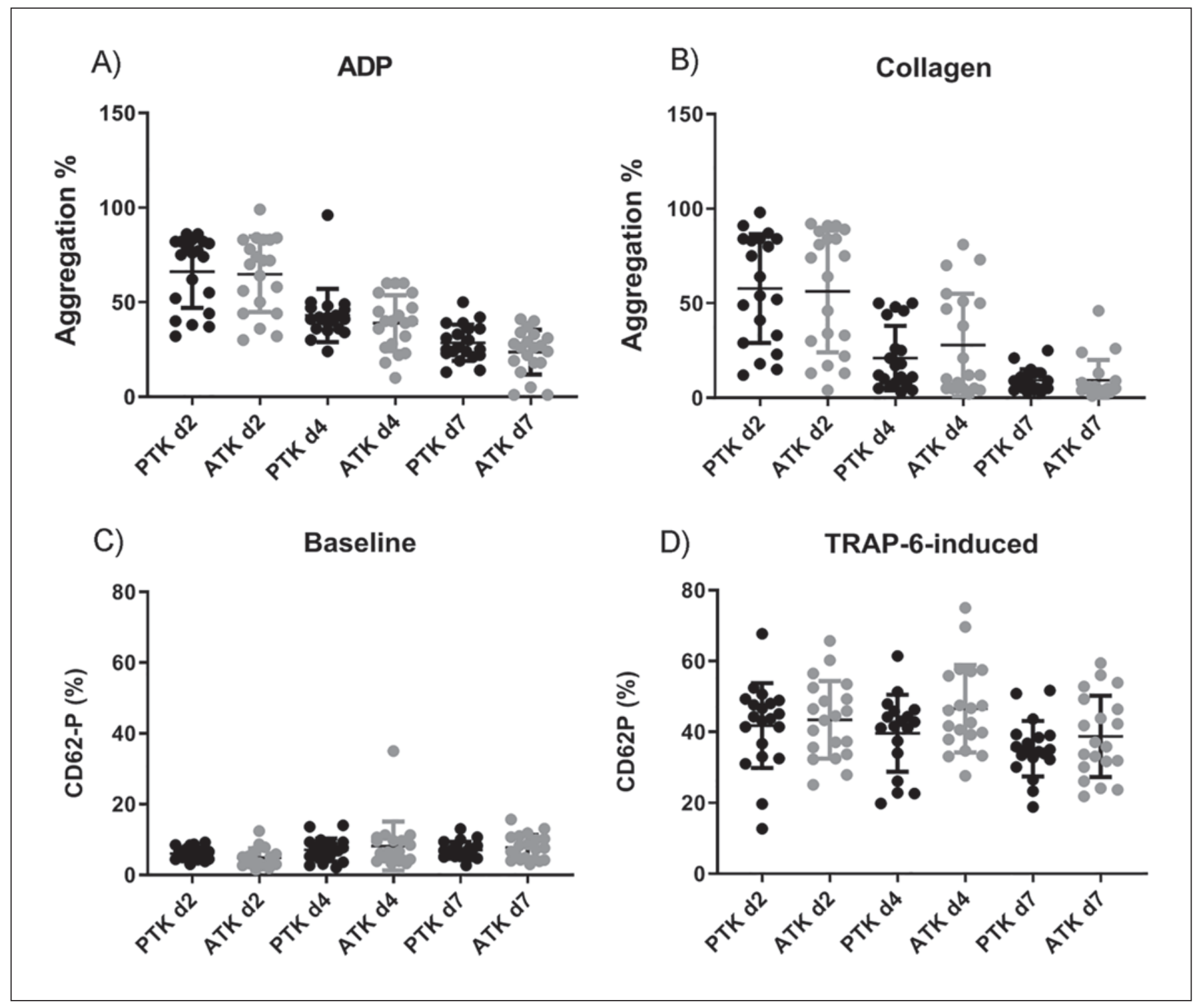

Fig. 3. Changes in PLT function during storage. PLT aggregation $\left(3 \times 10^{8} \mathrm{PLTs} / \mathrm{mL}\right)$ was tested with $10 \mu \mathrm{mol} / \mathrm{L}$ $\operatorname{ADP}(\mathbf{A})$ and $2 \mu \mathrm{g} / \mathrm{mL}$ collagen (B). PLT P-selectin (CD62-P) expression was determined by flow cytometry under baseline (C) and stimulated (10 $\mu \mathrm{mol} / \mathrm{L}$ TRAP-6) (D) conditions. d2, day 2; d4, day 4; d7, day 7 . Single values are presented with bars indicating the medians (1st and 3rd quartiles); $n=20$ PPCs (black dots), $n=20$ APCs (gray dots).

were below $12 \%$ at all times (Fig. 3C). The inducibility of P-selectin expression by TRAP-6 was very heterogeneous, and median values on days 2 and 4 amounted to $45 \%$ using $10 \mu \mathrm{mol} / \mathrm{L}$ TRAP-6 for PPCs and APCs, and declined by $10-15 \%$ until day 7 . A broad heterogeneity was observed, regardless of the PC type (Fig. 3D). The low sP-selectin levels in supernatant plasma (Table 1) and the moderate changes during storage are all in line with a well-preserved responsiveness of PLT P-selectin expression in both types.

Annexin V binding to PLTs and microvesicles was used as a measure of apoptosis-related PS expression. During the whole storage period, all binding values were below $1 \%$, indicating that there was no significant apoptosis, either in PPCs or in APCs (data not shown).

\section{Effect of Shear Stress on PLT Adhesion and Activation}

To evaluate the effect of shear forces on PLT stability, PLT suspensions were exposed to wall shear stress (shear rate $1,700 \mathrm{~s}^{-1}$ ) on their passage through the collagen-coated channel slides (Fig. 4A). The number of adherent PLTs was low under static conditions and in the presence of shear forces. The addition of $5 \mu \mathrm{mol} / \mathrm{L}$ TRAP- 6 increased the number of adherent PLTs in PPCs and APCs by approximately 2 -fold under static and 1.5-fold under shear stress conditions on day 2 . On day 4 , the level of adherent 
Fig. 4. Effect of shear stress on PLT adhesion and aggregation. PPCs and APCs adjusted to $3 \times 10^{8} \mathrm{PLTs} / \mathrm{mL}$ were perfused over the collagen surface of ibidi $\mu$-slides ${ }^{\mathrm{TM}}$ at wall shear rates of $1,700 \mathrm{~s}^{-1}\left(4 \mathrm{~min}, 37^{\circ} \mathrm{C}\right)$ or left static, both in the absence and presence of $5 \mu \mathrm{mol} / \mathrm{L}$ TRAP- 6 . Adherent PLTs, stained with anti-CD41-FITC were analyzed by fluorescence microscopy. Magnification, $\times 630$. A Representative images of PLT adhesion are shown for PPC (left) and APC (right) after 2 days (top) and 4 days (bottom) of storage in the presence of shear stress and TRAP-6. Scale bars, $20 \mu \mathrm{m}$. B Numbers of adherent PLTs were evaluated on 10 micrographs per condition and PC. Bars indicate the medians (1st and 3rd quartiles). $n=10$ PPCs, $n=10$ APCs. C Aggregation responsiveness to $2 \mu \mathrm{g} / \mathrm{mL}$ collagen of non-adherent PLTs pre- and postchallenge. $n=9$ PPCs (black dots), $n=10$ APCs (gray dots).
(A)
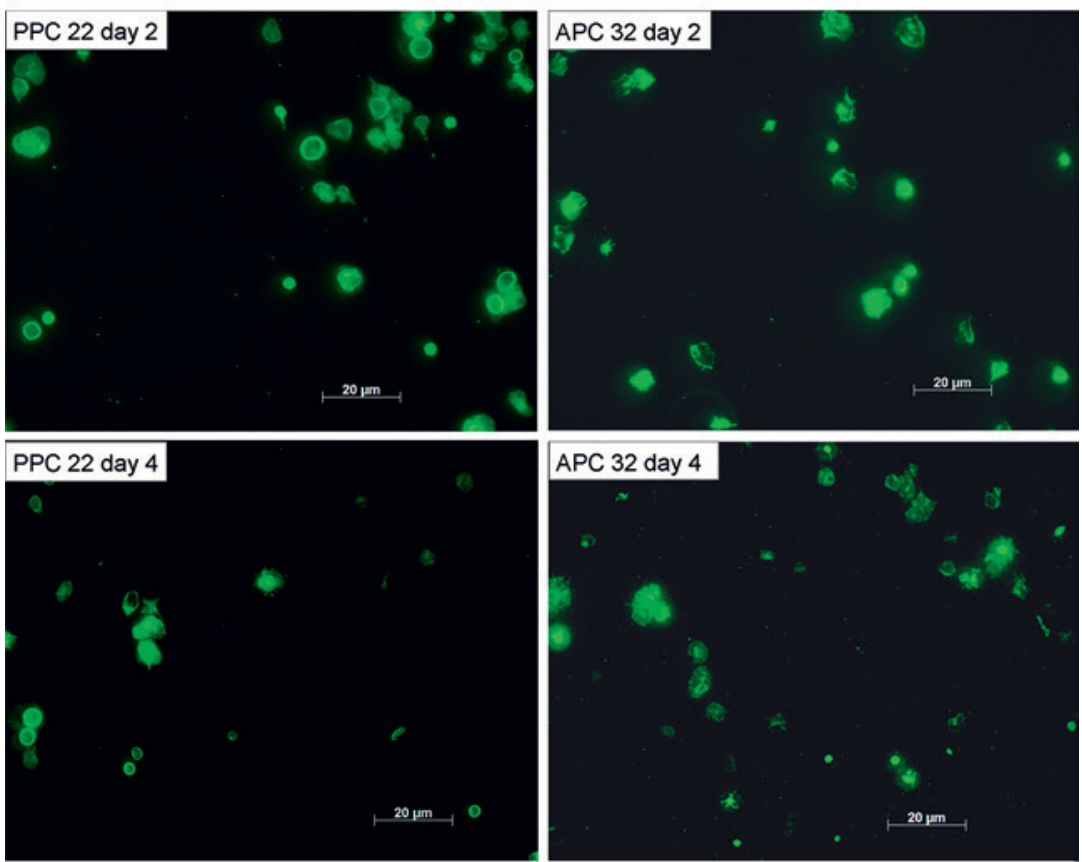

(B)
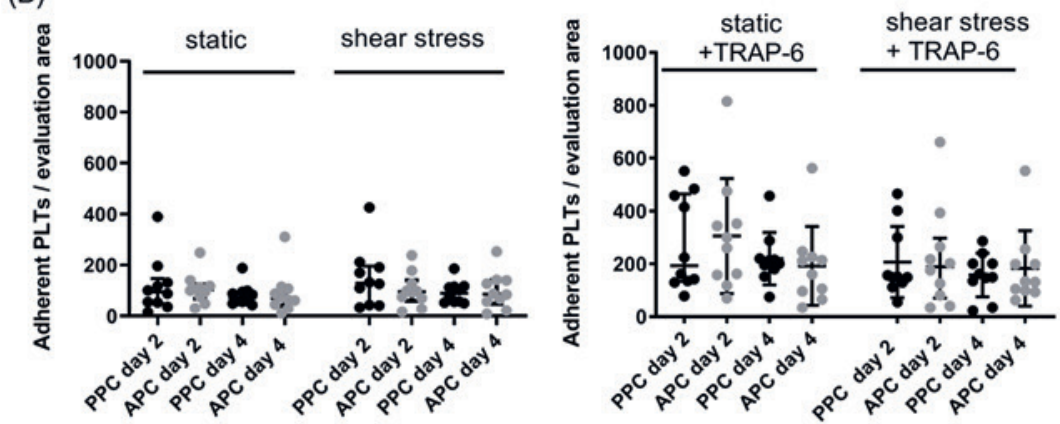

(C)
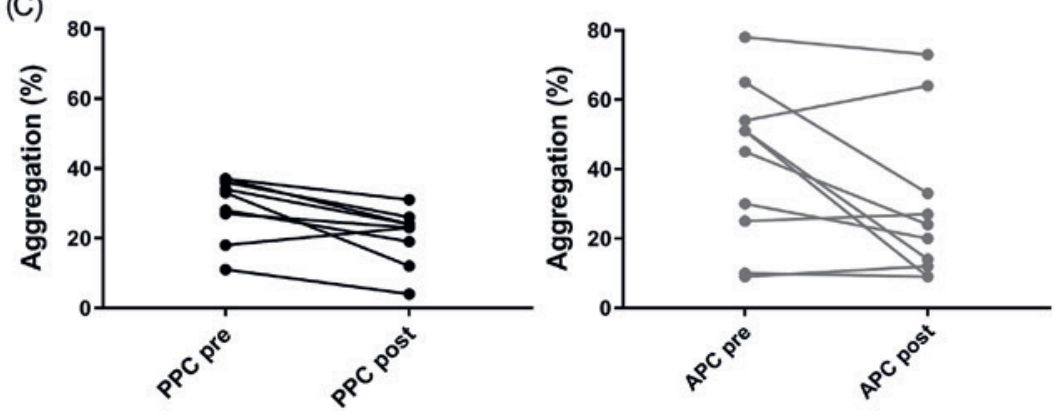

PLTs decreased by $20-30 \%$ (Fig. 4B). There was no significant difference between PPCs and APCs.

To examine stress-related alterations in the non-adherent PLT fraction and in the supernatant plasma, we performed additional experiments on day 3 of storage by applying static (pre-challenge) and flow conditions with elevated wall shear stress ( shear rate $1,700 \mathrm{~s}^{-1}$ ) in the presence of TRAP-6 (post-challenge) (Table 3). Constant plasma LDH levels and the PS expression below 2\% indicated an equally preserved PLT stability in both PC types, even after stress treatment. P-selectin expression of the non-adherent PLTs was largely unchanged; however, 2 of 19 PC samples presented strong increases in P-selectin levels. Similarly, the release of sP-selectin and CXCL-7/ NAP-2 in individual samples indicated a heterogeneous pattern of reactivity (Table 3 ). More important were the 
Table 3. Parameters for PLT viability and activation before and after a challenge by shear forces and TRAP-6

\begin{tabular}{|c|c|c|c|c|}
\hline \multirow[t]{2}{*}{ Parameters } & \multicolumn{2}{|l|}{$\operatorname{PPC}(n=9)$} & \multicolumn{2}{|l|}{$\mathrm{APC}(n=10)$} \\
\hline & pre & post & pre & post \\
\hline LDH, IU/l & $198(146 ; 260)$ & $187(166 ; 222)$ & $195(151 ; 223)$ & $186(123 ; 232)$ \\
\hline PLT P-selectin expression, \% & $10.6(8.1 ; 15.5)$ & $8.5(6.5 ; 11.6)$ & $7.7(1.7 ; 18.9)$ & $10.6(5.6 ; 17.5)$ \\
\hline sP-selectin, ng/mL & $4.6(-4.6 ; 17)$ & $11.4(6.8 ; 22.3)$ & $0.1(-8.1 ; 8.6)$ & $23.1(10.0 ; 35.2)$ \\
\hline CXCL7/NAP2, ng/mL & $657(304 ; 1,536)$ & $1,579(845 ; 1,924)$ & $255(71 ; 1,733)$ & $1,680(905 ; 3,177)$ \\
\hline \multicolumn{5}{|l|}{ Aggregation, \% } \\
\hline ADP & $48(37 ; 66)$ & $54(25 ; 70)$ & $52(27 ; 66)$ & $37(14 ; 60)$ \\
\hline Collagen & $33(11 ; 37)$ & $23(4 ; 31)$ & $48(9 ; 78)$ & $22(9 ; 73)$ \\
\hline
\end{tabular}

PLTs $\left(3 \times 10^{8} / \mathrm{mL}\right)$ were analyzed before (pre) and after (post) challenge by shear stress and TRAP- 6 on day 3 of storage, as described in Materials and Methods. sP-selectin and CXCL7/NAP-2 are expressed as the difference between $\mathrm{AB}$ plasma and the tested PLT suspensions. Values are given as medians (with minima and maxima). LDH, lactate dehydrogenase; sP-selectin, soluble P-selectin.

alterations in the PLT responsiveness in aggregation, which, in individual samples, manifested as a substantial loss of sensitivity to ADP (Table 3 ) and collagen (Fig. 4C). These functional alterations were observed in individual PCs of both types (PPC and APC).

\section{Discussion}

PCs are well-established therapeutic products in transfusion medicine that principally differ in their manufacturing process as pool or apheresis PCs. As the possible differences between PPCs and APCs have not yet been comprehensively studied, this study was designed to compare the in vitro quality and functional equivalence of the two PC types. To avoid heterogeneity due to different compositions of the PLT matrix with respect to the types and proportions of additive solutions, we decided to confine this study to PPCs and APCs produced in $100 \%$ plasma. Based hereon, further studies would be needed to determine PLT functionality in plasma-reduced and pathogen-inactivated preparations, the results of which should best be supported by clinical data.

The analyzed concentrates varied in terms of PLT content, volume, and residual cell numbers within the specification limits in compliance with the German Hemotherapy Guidelines [21]. Balanced metabolic conditions were found in both PC types as indicated by almost identical glucose consumption and lactate production rates for all PPCs and APCs throughout the storage period and independent of the initial glucose concentration. Consistently, $\mathrm{pH}$ values moderately decreased and the plasma $\mathrm{LDH}$ concentrations only slightly increased. The PLT morphology, assessed by TEM on day 4, was mainly discoid, whereas degranulated or apoptotic cells were almost absent, which underlines the cell integrity and complies with earlier results [12]. This finding was also in line with classical optical assays (HSR, ESC, and swirling), which yielded consistently stable results during the entire period of storage.

To assess pre-activation and functional capacities we estimated spontaneous PLT P-selectin and the agonist inducible P-selectin expression. Low baseline P-selectin levels are consistent with a high TRAP-6 sensitivity of Pselectin expression for both PC types. PLT aggregation revealed functional differences and partial deficiencies. Individual PCs from both product types were insensitive to AA and only weakly sensitive to collagen stimulation on day 2. We detected this donor-related impairment in both PC types and suspect that it could be caused by cyclooxygenase inhibitor intake (e.g., aspirin ${ }^{\circledR}$ ), which possibly was not recognized in the anamnestic donor interview, as previously reported [22]. In addition, a progressive loss of aggregability on days 4 and 7 became evident, which was observed in PPCs and APCs to the same extent. These alterations, known as storage lesions, are consistent with earlier studies performed in 100\% plasma [23] and in plasma-reduced PCs [24], which reported comparable levels of pre-activated PLTs in plasma and increased spontaneous activation in additive solution. Additional information on PLT alterations was obtained from PLT constituents released from intracellular granules or shedding of extracellular receptor domains. The moderate increase in the released chemokines RANTES and CXCL7, as well as in the soluble receptor domains (sP-selectin and sCD40L), demonstrated a largely maintained integrity until day 4 of storage.

Conventional in vitro methods do not determine PLT stability under shear forces comparable to those in the human arterial vessel system [25]. Therefore, we tested PLT robustness and reactivity in the presence of shear 
forces in a fluidic system. The low baseline adhesiveness increased under stimulation with TRAP-6, even in the presence of shear forces. Analysis of challenge-induced changes in non-adherent PLTs and plasma revealed differential responses, comprising moderate activation and also marked functional deficiency, e.g., in aggregation. Overall, this approach revealed a challenge-induced moderate elevation of activation markers on PLTs and in the plasma of PPCs and APCs, irrespective of the product type. However, the low performance of individual concentrates raises questions about the cause, persistence, and possible quality-indicating value since a significant loss of aggregation response after challenge may indicate poor robustness.

Another quality issue concerns the content of bioactive peptides derived from PLTs and residual cells that accumulate under storage and can negatively affect transfusion success $[3,26]$ or even cause side effects. According to our data, elevated cytokine levels, such as high IL-6, CXCL7, and RANTES levels, were already found in individual PCs at the beginning of the storage period. A correlation with the manufacturing type was not evident; rather, donor-related factors are conceivable. Dietary habits, factors related to the lifestyle, smoking, or the glycemic index have been reported to impact PLT function [27], but in our study could not be ascribed to individual PCs because the preparations were obtained from routine blood banking without such donor-specific information. Through testing for the well-known effects of COX-1 inhibitor intake by use of AA [28], our data revealed individual functional deficits due to a probable medication. To investigate the impact of donor variables would require a larger survey with recording of donor characteristics and ideally studying repeated donations of each donor.

The reversibility and PLT recovery from storage lesions have been reported [29], emphasizing the need for further evaluation of those PCs that exhibited strong functional deficits after challenge. In addition, clinical studies are required to assess the informative value of this type of stress test. In a recent clinical study, $\mathrm{pH}$ value and lactate production rate were reported to significantly correlate with in vivo PLT recovery and survival [30]. In our investigation, constant $\mathrm{pH}$ values and equal lactate production rates indicate comparably good quality and function for PPCs and APCs.

In conclusion, in this study, the PCs produced from pooled buffy coats or by apheresis performed equally well in quality parameters and in vitro function. Data obtained with established assay systems and results from a microfluidic perfusion system, which challenged the conditions of elevated wall shear stress, yielded comprehensive data and demonstrated a comparable PLT stability in PPCs and APCs.

\section{Limitations}

This in vitro analysis of PLT function was designed to principally compare the impact of the manufacturing process on the quality of PPCs and APCs in plasma. The fact that the majority of PCs are plasma-reduced concentrates containing different additive solutions necessitates similar studies of this type. In addition, pre-analytical influences cannot be excluded, although all possible efforts have been made to avoid any bias due to transport, lack of movement, or oxygen supply.

\section{Acknowledgments}

We gratefully acknowledge the valuable technical assistance of Annette Rausch-Müller and Salvatore Volpe, who conducted PLT function testing and biochemical analyses; Regina Eberle, who conducted the TEM sample processing; Anett Abu Karim, who conducted the viscosity measurements; and Sylvia Rosenkranz, who conducted the LDH assays. We also wish to thank Anja Schneider and Dr. Marcel Prax for conducting the sterility testing and are grateful to Dr. Dr. Doris Oberle for her valuable discussion.

\section{Statement of Ethics}

The PCs were purchased from public blood banks. Ethical principles, donor informed consent, and anonymization were obeyed. The research was conducted ethically in accordance with the World Medical Association Declaration of Helsinki.

\section{Disclosure Statement}

The authors have no conflicts of interest to declare.

\section{Funding Sources}

This study was funded by the German Ministry of Health (grant ZMVi1-2515-FSB-778).

\section{Author Contributions}

Sarah A. Fiedler performed the research and data analysis, and wrote and revised the paper. Klaus Boller performed the electron microscopic research, analyzed and interpreted the data, and revised the paper. Ann-Christine Junker performed the research and analysis, and revised the paper. Christel Kamp carried out the statistical evaluation, interpreted the data, and critically revised the manuscript. Anneliese Hilger designed the study, interpreted the data, and critically revised the manuscript. Wolfgang Schwarz designed the study, interpreted the data, and critically revised the paper. Rainer Seitz designed the study, interpreted the data, and critically revised the paper. Ursula Salge-Bartels designed the study, performed acquisition and interpretation of data, and wrote and revised the paper. All authors critically reviewed the manuscript and agreed upon submission. 


\section{References}

1 Slichter SJ, Davis K, Enright H, Braine $\mathrm{H}$, Gernsheimer T, Kao KJ, et al. Factors affecting posttransfusion platelet increments, platelet refractoriness, and platelet transfusion intervals in thrombocytopenic patients. Blood 2005 May; 105:4106-14.

2 Stanworth SJ, Navarrete C, Estcourt L, Marsh J. Platelet refractoriness-practical approaches and ongoing dilemmas in patient management. Br J Haematol. 2015 Nov;171(3):297305.

3 Aubron C, Flint AW, Ozier Y, McQuilten Z. Platelet storage duration and its clinical and transfusion outcomes: a systematic review. Crit Care. 2018 Aug;22(1):185.

4 Kelly AM, Garner SF, Foukaneli T, Godec TR, Herbert N, Kahan BC, et al. The effect of variation in donor platelet function on transfusion outcome: a semirandomized controlled trial. Blood. 2017 Jul;130(2):214-20.

5 Böck M, Rahrig S, Kunz D, Lutze G, Heim MU. Platelet concentrates derived from buffy coat and apheresis: biochemical and functional differences. Transfus Med. 2002 Oct; 12(5):317-24

6 Schrezenmeier H, Seifried E. Buffy-coat-derived pooled platelet concentrates and apheresis platelet concentrates: which product type should be preferred? Vox Sang. 2010 Jul; 99(1):1-15.

7 Dumont LJ, Szczepiorkowski ZM. Pooled platelet concentrates or apheresis platelets? N Engl J Med. 2013 May;368(19):1848-9.

8 Eriksson L, Kristensen J, Olsson K, Bring J, Högman CF. Evaluation of platelet function using the in vitro bleeding time and corrected count increment of transfused platelets. Comparison between platelet concentrates derived from pooled buffy coates and apheresis. Vox Sang. 1996;70(2):69-75.

9 Turner CP, Sutherland J, Wadhwa M, Dilger $\mathrm{P}$, Cardigan R. In vitro function of platelet concentrates prepared after filtration of whole blood or buffy coat pools. Vox Sang. 2005 Apr;88(3):164-71.

10 Brown CH 3rd, Lemuth RF, Hellums JD, Leverett LB, Alfrey CP. Response of human platelets to sheer stress. Trans Am Soc Artif Intern Organs. 1975;21:35-9.

11 Sheriff J, Bluestein D, Girdhar G, Jesty J. High-shear stress sensitizes platelets to subsequent low-shear conditions. Ann Biomed Eng. 2010 Apr;38(4):1442-50.
12 Neumüller J, Meisslitzer-Ruppitsch C, Ellinger A, Pavelka M, Jungbauer C, Renz R, et al. Monitoring of platelet activation in platelet concentrates using transmission electron microscopy. Transfus Med Hemother. 2013 Apr;40(2):101-7.

13 Kloft N, Neukirch C, Bobkiewicz W, Veerachato G, Busch T, von Hoven G, et al. Proautophagic signal induction by bacterial poreforming toxins. Med Microbiol Immunol (Berl). 2010 Nov;199(4):299-309.

14 Lombardo JF, Cusack NA, Rajagopalan C, Sangaline RJ, Ambruso DR. Flow cytometric analysis of residual white blood cell concentration and platelet activation antigens in double filtered platelet concentrates. J Lab Clin Med. 1993 Nov;122(5):557-66.

15 Schmitz G, Rothe G, Ruf A, Barlage S, Tschöpe $\mathrm{D}$, Clemetson KJ, et al. European Working Group on Clinical Cell Analysis: consensus protocol for the flow cytometric characterisation of platelet function. Thromb Haemost. 1998 May;79(5):885-96.

16 Dörmann D, Kardoeus J, Zimmermann RE, Kehrel B. Flow cytometric analysis of agonistinduced annexin $\mathrm{V}$, factor $\mathrm{Va}$ and factor $\mathrm{Xa}$ binding to human platelets. Platelets. 1998; 9(3-4):171-7.

17 Holme S, Moroff G, Murphy S; Biomedical Excellence for Safer Transfusion Working Party of the International Society of Blood Transfusion. A multi-laboratory evaluation of in vitro platelet assays: the tests for extent of shape change and response to hypotonic shock. Transfusion. 1998 Jan;38(1):31-40.

18 Fiedler SA, Junker AC, Seitz R, Salge-Bartels U. Effect of shear stress on platelet function and integrity. 51. Jahrestagung der Deutschen Gesellschaft für Transfusionsmedizin und Immunhämatologie (DGTI), Lübeck, 19-21 September, 2018. Abstracts. Transfus Med Hemother. 2018 Sep;45(Suppl 1):1-91.

19 Fuchs B, Budde U, Schulz A, Kessler CM, Fisseau C, Kannicht C. Flow-based measurements of von Willebrand factor (VWF) function: binding to collagen and platelet adhesion under physiological shear rate. Thromb Res. 2010 Mar;125(3):239-45.

20 Application note 11. Shear stress and shear rates for ibidi $\mu$-Slides - based on numerical calculations. ibidi $\mathrm{GmbH}, 2016$. Available from: https://ibidi.com/content/64-application-notes.
21 Bundesärztekammer, editor. Richtlinien zur Gewinnung von Blut und Blutbestandteilen und zur Anwendung von Blutprodukten (Richtlinie Hämotherapie): Aufgestellt gemäß $\$ \$ 12 \mathrm{a}$ und 18 Transfusionsgesetz von der Bundesärztekammer im Einvernehmen mit dem Paul-Ehrlich-Institut, Gesamtnovelle 2017. Köln: Deutscher Ärzte-Verlag; 2017.

22 Harrison P, Segal H, Furtado C, Verjee S, Sukhu K, Murphy MF. High incidence of defective high-shear platelet function among platelet donors. Transfusion. 2004 May;44(5): 764-70.

23 Cardigan R, Sutherland J, Garwood M, Bashir S, Turner C, Smith K, et al. In vitro function of buffy coat-derived platelet concentrates stored for 9 days in CompoSol, PASII or $100 \%$ plasma in three different storage bags. Vox Sang. 2008 Feb;94(2):103-12.

24 Singh S, Shams Hakimi C, Jeppsson A, Hesse C. Platelet storage lesion in interim platelet unit concentrates: A comparison with buffycoat and apheresis concentrates. Transfus Apheresis Sci. 2017 Dec;56(6):870-4.

25 Jilma-Stohlawetz P, Horvath M, Eichelberger B, Koren D, Jilma B, Panzer S. Platelet function under high-shear conditions from platelet concentrates. Transfusion. 2008 Jan;48(1): 129-35.

26 Sut C, Tariket S, Aubron C, Aloui C, HamzehCognasse $\mathrm{H}$, Berthelot $\mathrm{P}$, et al. The non-hemostatic aspects of transfused platelets. Front Med (Lausanne). 2018 Feb;5:42.

27 McEwen BJ. The influence of diet and nutrients on platelet function. Semin Thromb Hemost. 2014 Mar;40(2):214-26.

28 Temperilli F, Rina A, Massimi I, Montemari AL, Guarino ML, Zicari A, et al. Arachidonic acid-stimulated platelet tests: identification of patients less sensitive to aspirin treatment. Platelets. 2015;26(8):783-7.

29 Owens M, Holme S, Heaton A, Sawyer S, Cardinali S. Post-transfusion recovery of function of 5-day stored platelet concentrates. $\mathrm{Br}$ J Haematol. 1992 Apr;80(4):539-44.

30 Goodrich RP, Li J, Pieters H, Crookes R, Roodt J, Heyns AP. Correlation of in vitro platelet quality measurements with in vivo platelet viability in human subjects. Vox Sang. 2006 May;90(4):279-85. 